\title{
Smoothening of (001) and (111) Cu films epitaxially grown on Si substrates
}

\author{
Rosa Alejandra Lukaszew ${ }^{1}$, Ctirad Uher and Roy Clarke \\ Physics Department, University of Michigan, Ann Arbor, Michigan. \\ ${ }^{1}$ Presently at the Department of Physics and Astronomy, University of Toledo, Toledo, \\ Ohio.
}

\begin{abstract}
We report an in-situ study of the MBE growth of $\mathrm{Cu}$ films on hydrogen-terminated $\mathrm{Si}$ (001) and (7x7) reconstructed $\mathrm{Si}(111)$ substrates. Using correlated RHEED and STM data, we find a dramatic smoothing of epitaxial $\mathrm{Cu}(001)$ surfaces by annealing the asgrown films in the $120-160^{\circ} \mathrm{C}$ temperature range and somewhat less so for the $\mathrm{Cu}$ (111) films. Our measurements reveal a lower activation energy $(0.40 \pm 0.04 \mathrm{eV})$ for interterrace mass transport in $\mathrm{Cu}(001)$ than for $\mathrm{Cu}(111)(1.10 \pm 0.03 \mathrm{eV})$ the former possibly influenced by the presence of hydrogen. Scaling analysis of the subsequent $\mathrm{Cu}$ growth on the annealed smooth surfaces yields a coarsening exponent of $1 / 4$ for the (001) oriented films while this exponent is $1 / 3$ for the (111) films, providing for the first time experimental data for the same system in these two orientations.
\end{abstract}

\section{INTRODUCTION}

Buffer layers are often used to stabilize the growth of epitaxial films. In particular, $\mathrm{Cu}$ buffer layers grown on silicon substrates are used extensively for subsequent growth of epitaxial magnetic multilayers. Unfortunately the appearance of roughness as a result of noise, strain and step-edge barriers is an important challenge to the development of applications of epitaxial metal layers. In the case of heteroepitaxy, strain due to lattice mismatch and interfacial chemical reactions can also play an important role in roughening [1].

Interestingly, various reports indicate that copper can be grown at room-temperature as a single domain film on (001) $\mathrm{Si}-\mathrm{H}$ or (111)Si-7x7 surfaces, while it is not possible to grow single domain films on (111)Si-H or reconstructed (001)Si surfaces [2,3]. Thus, we describe here our results of an in-situ study comparing the mass transport kinetics of single domain (001) $\mathrm{Cu}$ films epitaxially grown on hydrogen terminated $\mathrm{Si}(100)$ and (111) $\mathrm{Cu}$ films epitaxially grown on $7 \times 7$ reconstructed $\mathrm{Si}(111)$, as well as the scaling behavior of growth parameters such as roughness and coarsening of the surface features.

In order to anneal epitaxial copper films to achieve smoother surfaces, the temperature should be high enough to provide enough energy to overcome step-edge barriers. However, as the $\mathrm{Cu}-\mathrm{Si}$ interaction is relatively strong, a p-d hybridization in the $\mathrm{Cu}-\mathrm{Si}$ intermixing region results in silicide formation, even at 100K [4]. The thickness of this silicide is strongly temperature dependent, thus limiting the annealing temperature range. In the following we will show that it is possible to obtain smooth single-domain copper films in both (001) and (111) orientations, grown on Si, via a mild annealing in a temperature range below predominant silicide formation. 


\section{EXPERIMENTAL}

Our studies were performed in a VG 80M molecular beam epitaxy (MBE) chamber equipped with an in-situ UHV (ultrahigh vacuum) scanning tunneling microscope (RHK model STM100) and a real-time reflection high-energy electron diffraction (RT-RHEED) imaging system [5]. Copper was evaporated from a K-cell at a rate of $0.2 \AA / \mathrm{sec}$. The films were grown under UHV conditions.

The (100) copper films were grown at room temperature (RT) on $\mathrm{Si}(001)$ substrates cleaned ultrasonically with acetone and alcohol for 15 minutes each and etched with HF ( $1 \%$ or $10 \%$ in volume) for 30 seconds. The surface of the substrates so treated is known to be hydrogen terminated. [6] Such surfaces are known to be inert for several minutes in air and for several hours in UHV at room temperature. This surface passivation is believed to be due to hydrogen termination of the dangling Si bonds, which renders the surface chemically stable.

The (111) copper films were grown at RT on (7x7) reconstructed (111) Si. The Si (111) substrates were prepared with the low temperature cleaning method proposed by Ishizaka and Shiraki [7]. This process consists of two steps: a) wet chemical treatment to etch the contaminated surface layers and to form a contamination-free passivative oxide film on the Si substrate, followed by b) heating under UHV conditions at temperatures below $900^{\circ} \mathrm{C}$ to remove the oxide film through thermal etching and obtain an atomically clean surface. The RHEED pattern for Si substrates treated in this way typically shows the (111) $7 \times 7$ superstructure, indicating an atomically clean surface.

\section{DISCUSSION}

\section{$\underline{\text { (001) Cu films }}$}

During the initial stages of growth, the RHEED pattern of silicon faded and, shortly thereafter, the crystalline structure of copper became apparent. The in-plane crystallographic axes of copper are rotated 45 degrees with respect to the silicon lattice as has been reported previously and ex-situ cross-sectional TEM and XRD corroborated the single domain quality of the (001) oriented films [6]. After $2000 \AA$ of $\mathrm{Cu}$ deposition, the RHEED pattern had a symmetrical spotty appearance characteristic of 3-D growth [Inset (a) in Figure 1].

The film was then annealed by increasing the temperature from room-temperature to $443 \mathrm{~K}$ (where the copper RHEED pattern fades) at a rate of $1 \mathrm{~K} / \mathrm{min}$. The heating process showed its effect through a sharpening of the copper streaks, indicating that the crystallinity improved significantly as a result of the annealing [Inset (b) in Figure 1].

A plot of 1/FWHM (full width at half maximum) of the specular RHEED streak vs temperature, shows two distinct regimes: A gradual narrowing for $\mathrm{T}<395 \mathrm{~K}$, and a more rapid evolution for $\mathrm{T}>395 \mathrm{~K}$. (Figure 1) We have also observed that for $\mathrm{T}>445 \mathrm{~K}$, the RHEED pattern becomes very diffuse indicating that the surface becomes rough again marking the onset of silicide formation. At approximately $470 \mathrm{~K}$ the copper RHEED pattern completely disappears. Subsequent films were annealed isothermally in the fast regime $(\mathrm{T}>390 \mathrm{~K}$ but below the silicide onset temperature, $\mathrm{T}<445 \mathrm{~K})$. Figure 2 (a) shows the STM image after growth at room temperature. The rms roughness of the surface is $\sim 70 \AA$ and the grain size is small $(\sim 160 \AA)$ due to interfacial strain (the lattice 
mismatch for an in-plane rotation of 45 degrees between the $\mathrm{Si}$ and $\mathrm{Cu}$ lattices is $~ 6 \%$ ) and limited diffusion kinetics that lead to deviations from ideal epitaxial growth. Figure 2 (b) on the other hand, corresponds to the STM image after annealing the film at $423 \mathrm{~K}$ for $4 \mathrm{hrs}$. The surface of the film now has large islands (average size $800 \AA$ ) and a mean rms roughness of $\sim 10 \AA$.

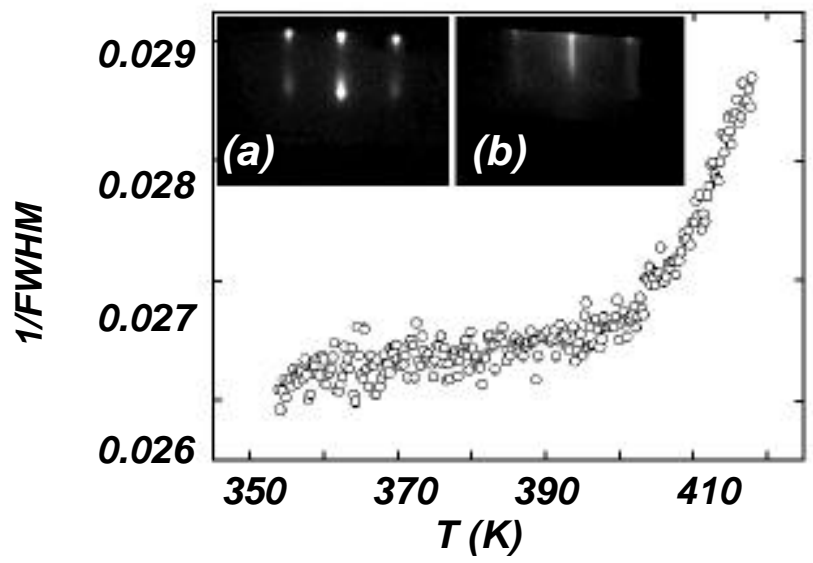

Figure 1. Plot of the inverse width (pixels) of the RHEED central streak as a function of temperature. Inset: RHEED images of a $200 \mathrm{~nm}$ thick $\mathrm{Cu}$ film, (a) as grown and (b) after annealing at $423 \mathrm{~K}$.
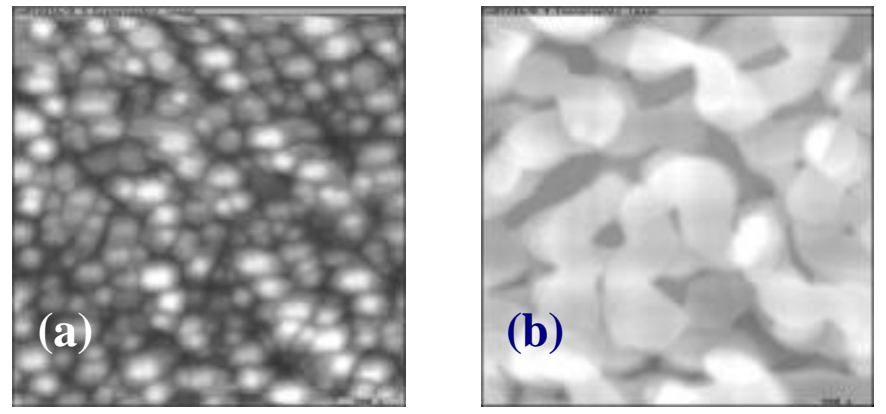

Figure 2. (a) STM image of the (001) Cu film as grown. (b) STM image of the same film after annealing. Both images are $300 \mathrm{~nm} \times 300 \mathrm{~nm}$ in size.

In order to analyze the smoothing kinetics of this system, we followed a similar analysis to that performed by Zuo et al. [8]. During the annealing process, the average terrace width on the film surface $\mathrm{L}(\mathrm{t})$ changes in time following a $\mathrm{t}^{1 / 3}$ law, consistent with kinetics driven by the line tension of curved step edges [9]. For T $>360 \mathrm{~K}$ the size of the initial terrace width $L\left(t_{0}\right)$ is not negligible and the $n=1 / 3$ growth law should read:

$$
\mathrm{L}^{3}(\mathrm{t})-\mathrm{L}^{3}\left(\mathrm{t}_{\mathrm{o}}\right)=\mathrm{A}(\mathrm{T})\left(\mathrm{t}-\mathrm{t}_{\mathrm{o}}\right)
$$


where $\mathrm{A}(\mathrm{T}) \propto(1 / \mathrm{T}) \exp \left[-\left(\mathrm{E}_{\mathrm{act}}\right) / \mathrm{k}_{\mathrm{B}} \mathrm{T}\right]$, is a temperature-dependent factor. The terrace size is inversely proportional to the width, $\Delta$, of the RHEED specular streak.

Thus, we plotted our RHEED data as $[1 / \Delta(\mathrm{t})]^{3}-\left[1 / \Delta\left(\mathrm{t}_{\mathrm{o}}\right)\right]^{3}$ vs $\left(\mathrm{t}-\mathrm{t}_{\mathrm{o}}\right)$. In such plots, the exponents for the time variable $\left[\left(\mathrm{t}-\mathrm{t}_{\mathrm{o}}\right)\right.$ in $\left.\mathrm{Eq}(1)\right]$ extracted for all temperatures were in the range $0.98 \pm 0.02$, consistent with the $n=1 / 3$ law. The pre-factor $A(T)$ was obtained for all temperatures from a least-squares fit to the data. An Arrhenius plot of $\ln [\mathrm{TA}(\mathrm{T})] \mathrm{vs} 1 / \mathrm{T}$ yielded an activation energy of $0.40 \pm 0.04 \mathrm{eV}$. The latest reported value for the activation energy for surface diffusion is $0.36 \pm 0.03 \mathrm{eV}$ [10]. Therefore, our value for the activation energy indicates that the fast smoothing of the surface is dominated by activated diffusion over step barriers in the temperature interval considered.

In order to further characterize the nature of the annealed $\mathrm{Cu} / \mathrm{Si}$ heteroepitaxial surface we studied the scaling behavior of the growth after further copper deposition. The rms roughness (interface width) $\xi$ for length $L$ and average thickness $\langle h\rangle$, for a system containing $\mathrm{N}$ sites with single valued heights $h_{i}$, is defined by the expression [11]:

$$
\xi\left(\mathrm{L},\langle h>)=\left[N^{-1} \sum_{i}^{N}\left[h_{i}-<h>\right]^{2}\right]^{1 / 2}\right.
$$

This theory predicts that when $\langle\mathrm{h}\rangle \rightarrow 0, \xi$ scales as:

$$
\xi(<h>) \propto\langle h\rangle^{\beta}
$$

where $\beta$ is the dynamic roughness exponent, and when $\langle h\rangle \rightarrow \infty$, it scales as:

$$
\xi(\mathrm{L}) \propto \mathrm{L}^{\alpha}
$$

where $\alpha$ is the static roughness exponent. The exponent $\beta$ was determined from a series of STM images obtained after the deposition of 3-15 ML (monolayers) of $\mathrm{Cu}$ on the smooth surface obtained after annealing. The exponent $\alpha$ on the other hand, was obtained from a single image obtained after $2000 \AA$ of further $\mathrm{Cu}$ deposition. Accordingly, $\alpha$ was derived from Eq. 4 where L corresponds to a segment of the STM line-scan measured along a direction parallel to the surface. The plot $\log \xi$ vs $\log \mathrm{L}$ exhibited a linear region for values of $\mathrm{L}$ smaller than the island size $\left(d_{s}\right)$ and after that it saturated. The slope of this straight line was found to be $1.03 \pm 0.03$. The exponent $\beta$ was evaluated from Eq. 3 . From a plot of $\log \xi$ vs $\log \langle h\rangle$, a straight line is obtained with a slope $\beta=0.25 \pm 0.01$.

We also measured the length-scale associated with coarsening. As $\langle h\rangle$ increases, the average island size $d_{s}$ increases according to:

$$
d_{s} \propto\langle h>\beta / \alpha
$$

From the plot $\log d_{s}$ versus $\log \langle h\rangle$ a value for $\beta / \alpha=0.25 \pm 0.01$ was obtained, and using the value obtained previously for $\alpha(\approx 1)$ we estimate $\beta=0.25$, consistent with the value derived from Eq. 3. Our values agree with those reported by Zuo et al. [8] for homoepitaxial growth of (001) copper at RT and also with theoretical models [12]. 


\section{(111) Cu films}

Figure 3 (a) shows the RHEED pattern of the $7 \times 7$ reconstruction of the (111) Si surface prior the growth. After $10 \mathrm{ML}$ of copper growth, the $7 \times 7 \mathrm{Si}(111)$ pattern has disappeared, and only the fundamental reflections of $\mathrm{Cu}$ can be observed [Figure 3 (b)]. Here the epitaxy is controlled by the formation of an interfacial compound with a thickness of a few monolayers. [3] The RHEED pattern shows only the fundamental reflections of the epitaxial $\mathrm{Cu}$ layers. This pattern has a spacing that is commensurate with the $\mathrm{Cu}$ lattice spacing and a unit cell that is not rotated with respect to the underlying Si substrate. After further deposition the RHEED pattern shows longer and sharper streaks, as determined by their smaller FWHM, indicating smooth single crystal film growth [Figure 3 (c)]. Figure 3 (d) shows the STM image of the surface as grown.
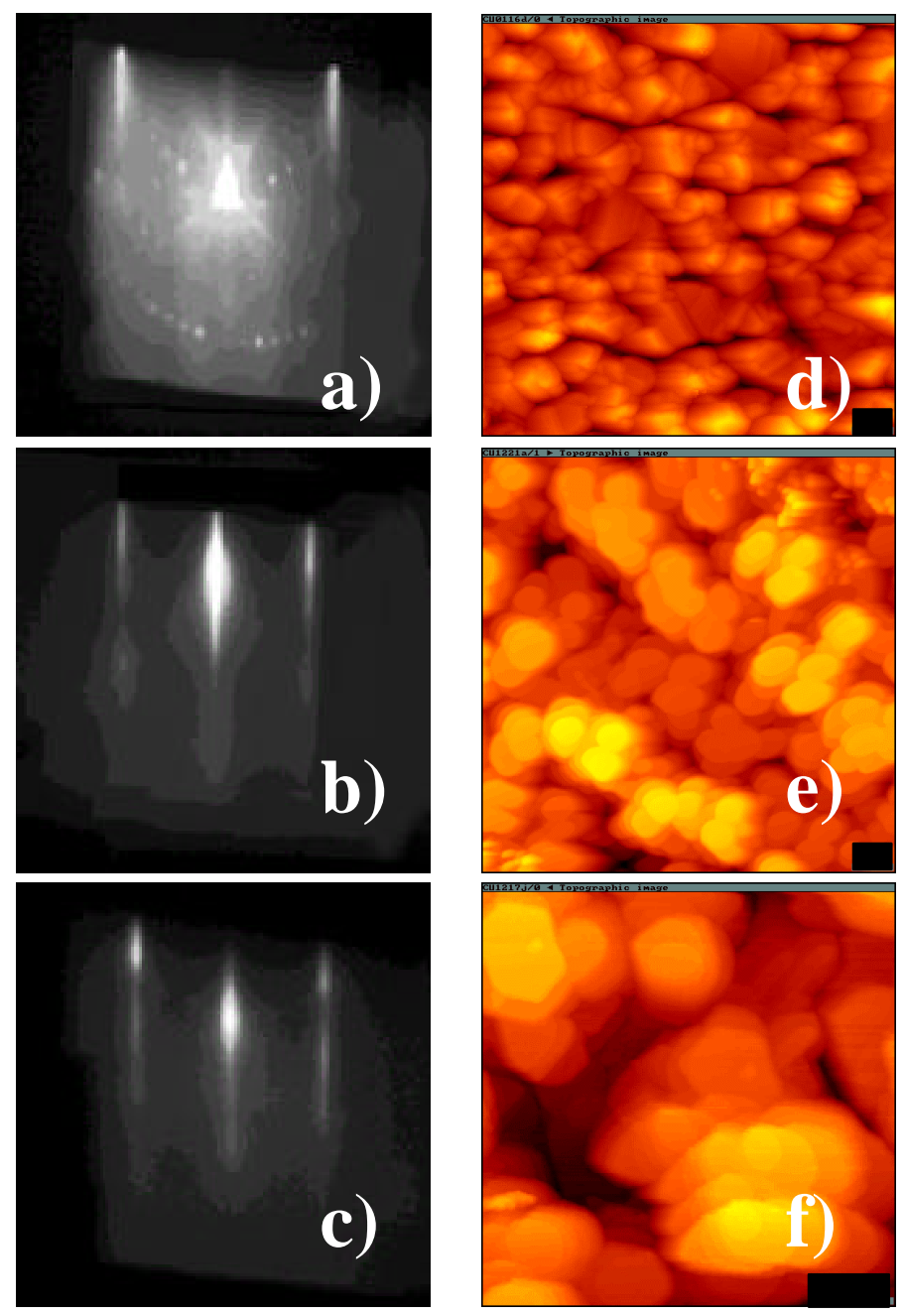

Figure 3. RHEED (left) and STM (right) images for (111) Cu films epitaxially grown/annealed on $7 \times 7$ (111) Si. Note: the scale-bar at the bottom right corner on the STM images corresponds to $50 \mathrm{~nm}$. 
The annealing of the films to improve the surface roughness had to be constrained even more than for the (001) case, as the film became rougher probably due to increase of the silicide region at the interface. For the temperature interval $298 \mathrm{~K}<\mathrm{T}<373 \mathrm{~K}$, the activation energy measured for the smoothening was $1.1 \mathrm{eV} \pm 0.03 \mathrm{eV}$

Despite this somewhat high activation energy for mass transport, a significant improvement of the surface roughness was observed [Figures 3 (e) and (f)]. Zuo et al. [8] also found an activation energy of $1.10 \mathrm{eV}$ in the temperature range $333 \mathrm{~K}<\mathrm{T}<368 \mathrm{~K}$ for (001) $\mathrm{Cu}$. The larger value measured for the activation energy for mass transport in $\mathrm{H}$ free systems, suggests that the $\mathrm{H}$ termination on the (001) silicon/copper interface may speed up the kinetics at the (001) $\mathrm{Cu}$ surface. Scaling studies of subsequent growth of copper on the (111) annealed surface yielded a coarsening exponent of 0.333 in agreement with theoretical predictions for the (111) orientation [13].

\section{CONCLUSIONS}

We have achieved smooth, epitaxial single-domain copper films MBE grown on Si substrates. The annealing temperature interval to smoothen the films is severely constrained by silicide formation at the interfaces, particularly critical for the (111) films. The activation energy measured for mass transport is considerably smaller for the (001) surface than for (111), suggesting that $\mathrm{H}$ termination on the (001) silicon/copper interface may speed up the kinetics at the (001) $\mathrm{Cu}$ surface. Scaling studies yield coarsening exponents of $1 / 4$ for the (001) surface and 1/3 for the (111) surface in agreement with theoretical models, and providing for the first time an experimental comparison of the growth for the same material and substrate.

\section{REFERENCES}

1. E. Han, E. Kampshoff, N. Wälchli, and K. Kern, Phys. Rev. Lett. 74, 1803 (1995); D. J. Eaglesham and M. Cerullo, Phys. Rev. Lett. 64, 1943 (1990).

2. $\quad$ R. A. Lukaszew, Y. Sheng, C. Uher, R. Clarke, Appl. Phys. Lett. 76, 724 (2000).

3. Z. H. Zhang, S. Haswgawa and S. Ino, Surf. Sci. 415, 363 (1998); T.I. M. Bootsma and T. Hibma, Surf. Sci.331-333, 636 (1995).

4. R. Padyath, J. Seth, S. V. Babu, and L. J. Matienzo, J. Appl. Phys. 75, 2326 (1993).

5. D. Barlett, C. W. Snyder, B. G. Orr, and R. Clarke, Rev. Sci. Instrum. 62, 1263 (1991); data acquisition using KSA400, k-Space Assoc. Inc., Ann Arbor, MI 48109.

6. B. Demczyk, R. Naik, G. Auner, C. Kota and U. Rao, J. Appl. Phys. 75, 1956 (1994).

7. A. Ishizaka and Y. Shiraki, J. Electrochem. Soc. 133, 666 (1986).

8. J. -K. Zuo and J. F. Wendelken, Phys. Rev. Lett. 70, 1662 (1993).

9. J. Villain, Europhys. Lett. 2, 521 (1986); A. Rettori and J. Villain, J. Phys. (Fr.) 49, 257 (1988).

10. H. Dürr, J. F. Wendelken, and J. -K. Zuo, Surf. Sci. 328, L527 (1995).

11. F. Family, Physica A. 168A, 561 (1990).

12. M. Siegert and M. Plishcke, Phys. Rev. Lett. 73, 1517 (1994).

13. J. Amar, Phys. Rev. B 60, R11317 (1999). 